\title{
Der erste ,griechische Kaiser“. Überlegungen zum Scheitern des Procopius Anthemius im Weströmischen Reich
}

\section{Dirk Henning}

\section{Einleitung}

Im Jahre 1959 veröffentlichte der Romancier und Essayist Frank Thiess (1890-1977) in einem Hamburger Verlag ein Sachbuch, in dem er die vermeintlich „dunklen Jahrhunderte“ des Oströmischen Reiches zwischen dem Tode Justinians I. im Jahre 565 und dem Regierungsantritt Leons III. im Jahre 717 darstellte. Thiess wählte für sein Buch den Titel „Die griechischen Kaiser", um auf diese Weise die seiner Meinung nach weitgehend unbekannte Tatsache herauszustellen, daß es sich bei dem griechisch geprägten, mittelalterlichen Reich von Byzanz um eine direkte Fortsetzung des Imperium Romanum der Antike handelte. ${ }^{1}$

Wohl ohne es zu ahnen, konfrontierte der Autor seine Leser so mit einem der wirkungsmächtigsten staatstheoretischen Probleme des Mittelalters, nämlich dem „Zwei-Kaiser-Problem“, dem Streit zwischen Byzanz und dem Westen um das Erbe des zerfallenen Römischen Weltreiches und um das damit verbundene Kaisertum. Byzanz konnte in dieser Auseinandersetzung zwar auf eine unanfechtbare staatsrechtliche Kontinuität verweisen, blieb jedoch propagandistisch in einer Defensivposition, da es die unmittelbare Herrschaft über die Keimzelle des einstigen Imperium, die Stadt Rom und

1 F. Thiess, Die griechischen Kaiser. Die Geburt Europas, Hamburg/Wien 1959. Zu Person und Werk des zu Lebzeiten heftig umstrittenen, heute jedoch fast in Vergessenheit geratenen Autors siehe nun Y. Wolf, Frank Thiess und der Nationalsozialismus (Unters uchungen zur deutschen Literaturgeschichte 114), Tübingen 2003. 
Italien, früh verlor und sich in der Folge zu einem völlig gräzisierten Staatswesen entwickelte. ${ }^{2}$

Um so energischer indes beharten die Kaiser in Konstantinopel auf ihrem „Römertum“, das seit dem frühen 9. Jahrhundert, in Reaktion auf die Kaiserkrönung Karls des Großen im Westen, sogar Eingang in ihre Titulatur fand. Als Basileis ton Romaion, als „Kaiser der Römer", bezeichneten sich die byzantinischen Herrschet bis 1453 ostentativ. Die intellektuelle Oberschicht Konstantinopels pflegte klassisches Griechisch und verdammte Latein als Barbarensprache, wies zugleich aber jeden Zweifel am eigenen Römertum entrüstet von sich. Hätte Frank Thiess den Titel seines Buches „Die griechischen Kaiser" 1000 Jahre zuvor in Konstantinopel formuliert, der Verlust zumindest der Nasenspitze wäre ihm sicher gewesen.

\section{Problemstellung}

Dieser scheinbar paradoxe, mittelalterliche Streit um Griechen- und Römertum reicht zurück bis in die Spätantike, in die Zeit der Auflösung der Westhälfte des Imperium Romanum im späten 5. Jahrhundert. Mit dem Tode Kaiser Valentinians III. erlosch 455 das letzte bedeutende Kaiserhaus, das beide Reichshälften regiert hatte, nämlich die theodosianische Dynastie. Es folgte im Weströmischen Reich eine Phase von Machtkämpfen und Bürgerkriegen, an der sich schon bald die auf Reichsboden siedelnden germanischen Völker beteiligten. Innerhalb zweier Jahrzehnte wurden so insgesamt neun Kaiser erhoben und wieder gestürzt. Am Ende stand der territoriale Zerfall des Westreiches, die Eliminierung des Kaisertums und die Machtübernahme in Italien durch das germanisch dominierte Militär. ${ }^{3}$

$\mathrm{Zu}$ den zahlreichen ephemeren Herrschergestalten, die während dieser Krise um die Macht kämpften, gehörte auch ein Oströmer, nämlich der aus Konstantinopel stammende und mit Unterstützung des Ostkaisers Leo I. nach Italien entsandte Procopius Anthemius. Anthemius gehörte den prominentesten Kreisen Konstantinopels an, besaß verwandtschaftliche Beziehungen sowohl zur konstantinischen wie zur theodosianischen Dynastie und

2 Überblicksdarstellung zuletzt bei H. H. Anton, Zweikaiserproblem, Lexikon des Mittelalters 9, 1999, 720-723 (mit älterer Literatur).

3 Zum folgenden sei auf meine Monographie Periclitans res publica. Kaisertum und Eliten in der Krise des Weströmischen Reiches 454/5-493 n.Chr. (Historia. Einzelschriften 133), Stuttgart 1999, verwiesen. Da sich dort umfassende Quellenbelege und Literaturangaben finden, ist die Dokumentation hier auf das Nötigste beschränkt worden. 
hätte im Jahre 457 um ein Haar den östlichen Thron bestiegen. Zehn Jahre später versuchte er sein Glück erneut, diesmal im Westen. Tatsächlich gelang es Anthemius, sich hier mehr als fünf Jahre lang, von 467 bis 472 , zu behaupten. Am Ende scheiterte aber auch er wie nahezu sämtliche seiner Vorgänger und Nachfolger in dieser Spätphase. Unter den dramatischen Begleitumständen einer Armeerevolte mit Belagerung und Eroberung Roms wurde Anthemius durch den germanischen Heermeister Ricimer gestürzt und ermordet. ${ }^{4}$

In einem Punkt allerdings unterschied sich das Schicksal des Anthemius von demjenigen der anderen Herrschergestalten seiner Zeit: Wie einige Quellen behaupten, wurde von den Gegnern des Kaisers im Kampf um die Macht die griechische Herkunft des Anthemius thematisiert, dabei sogar ein Gegensatz zwischen Graecus und Romanus konstruiert, mit dem Ziel, die Autorität des Kaisers zu beschädigen. In der Forschung hat sich einzig John Michael O'Flynn in einem kurzen Artikel aus dem Jahr 1991 mit diesem Phänomen beschäftigt. Er hält diese Quellenaussagen für glaubhaft und folgert aus ihnen, daß bereits zu dieser Zeit eine tiefgreifende Entfremdung zwischen Ostund Westrom erfolgt sei, daß die führenden gesellschaftlichen Kräfte des Westreiches schon im späten 5. Jahrhundert in den Eliten des Ostreiches nicht mehr Römer, sondern Griechen erblickt hätten, ja noch schlimmer: Fremde, die ihnen ferner standen als die germanischen Barbaren ihrer unmittelbaren Umgebung. Diese Kluft zwischen Ost und West, so O'Flynn weiter, sei für das Scheitern des Anthemius in Italien ursächlich gewesen. Es führe von ihr eine Kontinuitätslinie bis hinein ins hohe Mittelalter, zum eingangs geschilderten Zwei-Kaiser-Problem des 9. bis 13. Jahrhunderts. ${ }^{5}$

Die Quellenbasis, auf die O'Flynn seine weit ausgreifende These stützt, ist allerdings recht dünn und, wie sich zeigen wird, brüchig. Der historische Kontext ihrer Entstehung, aber auch der Regierungszeit des Anthemius selbst, bleibt weitgehend unberücksichtigt. Das Ziel der folgenden Ausführungen ist es deshalb, an diesen Punkten anzusetzen und O'Flynns These einer entsprechenden Prüfung zu unterziehen. Letztlich geht es dabei um die Frage, ob ein Zusammenhang - und wenn ja, in welchem Maße - zwischen der östlichen Herkunft des Anthemius und seinem Scheitern im Weströmischen Reich bestand, oder, zugespitzt formuliert, ob Anthemius Thron und Leben verlor, weil er ein ,griechischer Kaiser" war.

4 Person und Schicksal des Anthemius: Henning, Krise (wie Anm. 3), 42-46.

5 J. M. O'Flynn, A Greek on the Roman throne: the fate of Anthemius, Historia 40, 1991, 122-128. O'Flynns Thesen wurden jüngst weitgehend unkritisch übernommen von $P$. MacGeorge, Late Roman Warlords, Oxford/New York 2002. 


\section{Schriftquellen}

Zwei Quellen geben Hinweise darauf, daß die griechische Herkunft des Anthemius in der Auseinandersetzung des Kaisers mit seinen innenpolitischen Gegnern eine Rolle spielte:

Magnus Felix Ennodius, Bischof von Pavia zur Zeit des Königs Theoderich, berichtet in der von ihm verfaßten Vita seines Amtsvorgängers Epiphanius über dessen diplomatisches Geschick im Umgang mit den Mächtigen seiner Zeit. ${ }^{6}$ Epiphanius wurde aufgrund seiner Fähigkeiten in den 470er Jahren insgesamt dreimal mit heiklen Vermittlungsmissionen zwischen verfeindeten Parteien betraut. So vertrat er im Jahre 475 die Interessen des Kaisers Iulius Nepos bei den Westgoten und um 477 die ligurische Senatsaristokratie vor Odoaker. Bereits 471 hatte er im Auftrag des Heermeisters Ricimer und der ligurischen Provinzialversammlung versucht, eine Beilegung des Konfliktes zwischen Ricimer und Kaiser Anthemius zu erreichen. Dieser Vermittlungsversuch wird von Ennodius zwar als erfolgreich dargestellt, scheiterte aber in Wahrheit.

Für unser Thema von Bedeutung ist die Vorgeschichte dieser Mission, nämlich die Suche nach einem geeigneten Vermittler. Ennodius zufolge befragte Ricimer die ligurischen Senatoren mit den Worten: Quis est, qui Galatam concitatum revocare possit et principem? („Wer ist es, der den aufgeregten Galater und Princeps zur Besinnung bringen kann?") Die Provinzialversammlung schlägt daraufhin Bischof Epiphanius von Pavia vor, quem venerari possit quicumque, si est catbolicus et Romanus, amare certe (...) et Graeculus („den jeder verehren kann, wenn er ein Katholik und Römer ist, und den gewiß sogar ein Griechlein lieben kann"). ${ }^{7}$ Alle diese Schmähungen beziehen sich auf Anthemius, der hier als Galata concitatus und Graeculus verunglimpft und in direkten Gegensatz zu einem catholicus et Romanus gesetzt wird, dem also sowohl Rechtgläubigkeit wie auch Römertum abgesprochen werden.

6 Zu Ennodius und der Epiphanius-Vita zuletzt A. Gillett, Envoys and Political Communication in the Late Antique West, 411-533, Cambridge 2003, 148-171; S. A. H. Kennell, Magnus Felix Ennodius. A Gentleman of the Church, Michigan 2000, passim. Unersetzt ist nach wie vor M. Cesa, Ennodio. Vita del beatissimo Epifanio vescovo della chiesa $\mathrm{Pa}$ vese (Biblioteca di Athenaeum 6), Como 1988.

7 Ennod. V. Epiph. 53-55. - Die drastischen Formulierungen des Textes werden in seiner bislang einzigen deutschen Übersetzung durch M. Fertig, Magnus Felix Ennodius und seine Zeit. 2. Abschnitt: Vita S. Epiphanii, in: Jahres-Bericht über die KöniglichBayerische Studien-Anstalt zu Landshut in Niederbayern für das Schuljahr 1859/60, Landshut 1860,5 , nicht adäquat wiedergegeben. 
Zweifel an der Authentizität dieser von Ennodius wiedergegebenen Aussagen drängen sich deshalb geradezu auf. Wir erleben hier einen eklatanten Fall von Majestätsbeleidigung, begangen durch führende gesellschaftliche Kräfte des Westreiches gegenüber ihrem regierenden Souverän, und dies angeblich während der Vorbereitung einer diplomatischen Friedensmission zu eben diesem Souverän. Schon dieses Faktum spricht gegen die Glaubwürdigkeit der Darstellung. Ennodius war sicher bis zu einem gewissen Grad über die Regierungszeit und das Schicksal des Anthemius informiert, und dieses Schicksal hatte - wie noch zu zeigen sein wird - auch Probleme des Kaisers mit seiner Herkunft sowie mit der römischen Kirche eingeschlossen. $\mathrm{Daß}$ Ennodius Jahrzehnte zurückliegende Äußerungen jedoch im Wortlaut gekannt haben sollte, ist - wie so oft im antiken Quellenbestand - auszuschließen. Wir können davon ausgehen, hier nicht den Originalton des Heermeisters Ricimer oder der ligurischen Senatoren, sondern vielmehr denjenigen des Ennodius selbst vor uns zu haben. Die „Vita Epiphanii“ entstand in den 490er Jahren, als unter dem Pontifikat des Papstes Gelasius (492-496) das seit 483 zwischen Rom und Konstantinopel bestehende, sogenannte Akazianische Schisma, die erste große Kirchenspaltung zwischen Ost und West, besondere Virulenz erlangte. Es ist gut vorstellbar, daß die damals im italischen Klerus verbreitete, heftige Ablehnung der oströmischen Positionen Ennodius bei seiner Schilderung die Feder führte. Das Bestreiten der Rechtgläubigkeit und des Römertums des Anthemius, das Ennodius der ligurischen Aristokratie in den Mund legt, paßt in der hier zum Ausdruck gebrachten Schärfe jedenfalls sehr viel besser in seine eigene Zeit als in diejenige des Anthemius. ${ }^{8}$

8 Bereits in der Amtszeit seines Vorgängers Felix III. (483-492) hatte Gelasius als Diakon maßgeblich am Ausbruch des Schismas zwischen Ost und West mitgewirkt. Während seines eigenen Pontifikates verschärfte sich der Ton zwischen Rom und Konstantinopel weiter. Unter dem Schutz des ostgotischen Königs Theoderich wagte erstmalig ein Papst eine offene Konfrontation mit dem Kaiser. Vom Höhepunkt des Schismas, aus dem Jahre 495, datiert ein Brief des Gelasius an die Bischöfe der Provinz Dardania, in dem der Papst explizit auf das Beispiel des Anthemius verweist: Anthemius habe während seiner Herrschaft in Rom Häretiker (sc. Makedonianer, s. unten) unterstützen wollen, sei vom damaligen Papst Hilarus aber gezwungen worden, dies zu unterlassen (Gelas. ep. 26, 11, ed. A. Thiel, Epistolae Romanorum pontificum genuinae et quae ad eos scripta sunt, Bd. 1: A S. Hilaro usque ad S. Hormisdam, Braunsberg 1868). Diese Unterstellung mangelnder Rechtgläubigkeit des Anthemius deckt sich auffallend mit den etwa zeitgleich entstandenen Formulierungen des Ennodius. - Zu Gelasius vgl. R. Schieffer, Gelasius I., Lexikon des Mittelalters 4, 1999, 1197; G. Händler, Die abendländische Kirche im Zeitalter der Völkerwanderung (Kirchengeschichte in Einzeldarstellungen, Bd. I, 5), Berlin 1987, 90f. Wie Kennell, Ennodius (wie Anm. 6), 206-208, betont, zeigt sich im Gesamt- 
Als zweiter Beleg für die Bedeutung der griechischen Herkunft des Anthemius gilt ein Brief des gallischen Senators und späteren Bischofs von Clermont Sidonius Apollinaris aus dem Jahre 469, also aus der Regierungszeit des Anthemius. ${ }^{9}$ Sidonius berichtet in diesem Schreiben über die Anklage und Verurteilung des ehemaligen gallischen Prätorianerpräfekten Arvandus wegen Hochverrats, die kurz zuvor in Rom stattgefunden hatte. Arvandus wurde des crimen laesae maiestatis für schuldig befunden aufgrund eines von ihm verfaßten Schreibens an den Westgotenkönig Eurich, in dem er diesen aufforderte, keinen Frieden mit dem, so wörtlich, graecus imperator zu schlieBen. ${ }^{10}$

Im Unterschied zur „Vita Epiphanii““ ist dieser Beleg zeitgenössisch und stammt von einem Augenzeugen, der sowohl Anthemius als auch Arvandus persönlich kannte und beide vor Ort in Rom erlebt hatte. Der Aussage des Sidonius Apollinaris kommt deshalb ein ungleich höheres Gewicht zu als derjenigen des Ennodius. Das hochverräterische Schreiben wurde während des Arvandus-Prozesses als Beweismittel vorgelegt, und Sidonius zufolge bestritt der Angeklagte zu keiner Zeit dessen Authentizität. Es ist deshalb davon auszugehen, daß Arvandus den Kaiser tatsächlich offen als graecus verunglimpft, ihm also das Römertum abgesprochen hatte.

Nicht statthaft hingegen erscheint es, mit O'Flynn diese Haltung für die weströmische Oberschicht insgesamt zu postulieren. Die Arvandus-Affäre war ein Einzelfall, der juristische Konsequenzen nach sich zog. Gerade die Tatsache, daß es Standeskollegen des ehemaligen Präfekten waren, die in Rom als Ankläger auftraten, belegt die Isolierung des Arvandus. Zwar verfolgten jene gallischen Aristokraten, die sich in den Besitz seines Schreibens gebracht hatten und es den Richtern präsentierten, vorrangig persönliche Motive. Dies ändert jedoch nichts an ihrer offiziell zur Schau gestellten Ablehnung des Schreibens und seiner Inhalte.

werk des Ennodius ein durchaus unterschiedliches Bild des griechischen Ostens, abhängig von Auftraggeber und Adressat des jeweiligen Werkes.

9 Sid. ep. 1, 7. Datierung des Briefes nach Helga Köhler, C. Sollius Apollinaris Sidonius. Briefe. Buch I. Einleitung-Text-Übersetzung-Kommentar, Heidelberg 1995; André Loyen, Sidoine Apollinaire, Bd. 2: Lettres (Livres I-V), Paris 1970.

10 Zum Arvandus-Prozeß: H. C. Teitler, Un-Roman activities in late antique Gaul: the cases of Arvandus and Seronatus, in: J. Drinkwater/H. Elton (Hgg.), Fifth-century Gaul: a crisis of identity?, Cambridge 1992, 309-317; Jill Harries, Sidonius Apollinaris, Rome and the barbarians: a climate of treason?, ebd., 306f.; dies., Sidonius Apollinaris and the Fall of Rome. AD 407-485, Oxford 1994, 92-94; D. Henning, Messius Phoebus Severus und die Chronologie der praefecti Urbi unter Kaiser Anthemius (467-472), ZPE 108, 1995, 154f.; ders., Krise (wie Anm. 3), 165 mit Anm. 255. 


\section{Historischer Kontext ${ }^{11}$}

Konstantinopel hatte im Verlauf des 5. Jahrhunderts wiederholt in die $\mathrm{Ge}$ schicke des Westreiches eingegriffen, verstärkt seit Ausbruch der dortigen Dauerkrise nach 455. Diese Eingriffe waren in Italien nicht immer begrüßt worden und hatten zu mancherlei Spannungen geführt. Der vom oströmischen Kaiser Leo I. unternommene Versuch, mit Anthemius einen eigenen Kandidaten für den westlichen Kaiserthron zu lancieren, stellte zweifellos den Höhepunkt der östlichen Einflußnahme dar. Daß es sich noch dazu um einen Kandidaten mit griechischer Muttersprache und ohne jede Beziehung in den Westen handelte, vereinfachte die Lage wohl kaum.

Auf der anderen Seite waren sich Leo und Anthemius dieser Problematik bewußt. Von Anfang an trafen sie Maßnahmen, um die Legitimität des Thronkandidaten zu unterstreichen, sein „Römertum“ hervorzuheben und seine Akzeptanz durch die westlichen Eliten zu befördern. Seit 466 verhandelte Leo mit den politisch relevanten Kräften des Westreiches, d. h. dem Senat und vor allem dem Heermeister Ricimer als dem eigentlichen Machthaber vor Ort, um den Boden für Anthemius zu bereiten. Anschließend wurde dieser noch im Osten zum Caesar erhoben, so wie einst Valentinian III., der letzte Kaiser, der von Konstantinopel aus nach Rom entsandt worden war. Die Parallele war durchaus beabsichtigt: In Italien eingetroffen, ließ sich Anthemius im Frühjahr 467 auf dem Landgut ad duas lauros zum Kaiser erheben, an jener Stelle also, an dem die weströmische Dauerkrise zwölf Jahre zuvor mit der Ermordung Valentinians III. ihren Anfang genommen hatte. Der neue Kaiser stellte sich damit gezielt in die Nachfolge des letzten Vertreters der theodosianischen Dynastie, die noch einmal beide Hälften des Imperium zusammengeführt hatte.

Die Idee der Reichseinheit, die er auf diese Weise beschwor, zog sich als roter Faden ebenso durch den Panegyrikus, den Sidonius Apollinaris am 1. Januar 468 anläßlich der Konsulatsübernahme des neuen Herrschers in Rom vortrug. Darüber hinaus versuchte Anthemius, sich gezielt in altrömische Traditionen zu stellen: Er residierte in Rom statt in Ravenna und ließ hier Münzen mit „klassischen“ Legenden wie SALVS REI PVBLICAE oder GLORIA REI PVBLICAE prägen. Wie zuletzt Valentinian III. führte der Kaiser Reparaturarbeiten am Kolosseum durch, ferner an Thermen und Aquädukten der Stadt Rom. Die Stellung des Senats erfuhr durch die Über-

11 Vgl. Henning, Krise (wie Anm. 3), passim. 
tragung des oben genannten Arvandus-Prozesses an ein senatorisches Standesgericht eine besondere Aufwertung.

Jedoch unterliefen Anthemius während seiner Regierungszeit auch Fehler, die durchaus in Zusammenhang mit seiner Herkunft standen. Der neue Kaiser traf in Italien mit einem umfangreichen oströmischen comitatus ein, dem zahlreiche Griechen und nicht wenige Heiden angehörten. Ein Heide, der in Alexandreia lebende Messius Phoebus Severus, wurde für 470 zum Konsul erhoben und avancierte anschließend zum Stadtpräfekten. Vergleichbares hatte die Stadt Rom seit sechzig Jahren nicht mehr erlebt. Ein weiterer bekennender Heide, der Dalmater Marcellinus, erhielt ein Heermeisteramt verliehen. Dem Griechen Philotheus schließlich, einem engen Freund des Anthemius und Anhänger der als häretisch verdammten Makedonianischen Glaubensrichtung, wurde in Rom die Einrichtung neuer Versammlungstäume (conciliabula nova) für "verschiedene Sekten" gestattet. ${ }^{12}$ Diese offen zur Schau gestellte, religiöse Toleranz des Kaisers provozierte heftigen Widerstand seitens der römischen Kirche und ihres Bischofs Hilarus.

Auch im Umgang mit der Senatsaristokratie bewies Anthemius keine glückliche Hand. Knapp 100 Jahre zuvor hatte in einer vergleichbaren Situation der aus Spanien gebürtige Kaiser Theodosius I. (379-395) vor der Aufgabe gestanden, seine Herrschaft im Ostreich durchzusetzen. Wie jüngst Hartmut Leppin hervorhob, war Theodosius - dem Außenseiter - die Gewinnung der Konstantinopolitaner Eliten durch die Verbindung mit einer ihrer „Schlüsselgestalten“, dem Redner Themistios, gelungen. ${ }^{13}$ Anthemius hingegen wählte 467 als seine „Schlüsselgestalt“ in Rom ausgerechnet den Gallier Sidonius Apollinaris, der gegenüber dem stadtrömischen Establishment ebenso eine Außenseiterposition einnahm wie er selbst, ja, den die römischen Senatoren elf Jahre zuvor an der Seite seines Schwiegervaters, des ephemeren Kaisers Avitus, aus der Stadt gejagt hatten. Diesem Sidonius Apollinaris wurde nun die Ehre zuteil, den Panegyrikus auf den Konsulatsantritt des neuen Kaisers halten zu dürfen. Anschließend wurde er zum Stadtpräfekten ernannt.

12 Philotheus: Gelas. Ep. 26, 11 (wie Anm. 8). - Heidentum des Marcellinus: Dam. V. Isid. Epit. Phot. $91+$ F 157-158, ed. C. Zintzen, Damascii vitae Isidori reliquiae, Hildesheim $1967=$ F 69A + D-E, ed. P. Athanassiadi, Damascius. The Philosophical History. Text with translation and notes, Athen - Oxford 1999; Marc. Com. s. a. 468, ed. Th. Mommsen, MGH AA XI, 89f. - Heidentum des Severus: Dam. Epit. Phot. $108=77$ Athanassiadi.

13 H. Leppin, Theodosius der Große - Auf dem Weg zum christlichen Imperium, Darmstadt 2003, 57-63. 
Mit den geschilderten Maßnahmen brüskierte Anthemius einen wichtigen Teil der weströmischen Eliten und konterkarierte zugleich seine Versuche, das eigene „Römertum“ zu beweisen. Ob für diese Fehler sein unterschiedlicher kultureller Hintergrund, mangelnde Vorbereitung oder einfach Unfähigkeit verantwortlich waren, sei dahingestellt. Auf jeden Fall dürfte Anthemius auf diese Weise ursprünglich vorhandene Ressentiments gegen seine griechische Herkunft vollauf bestätigt haben. ${ }^{14}$

Und dennoch: Es existieren keine Hinweise für eine hieraus resultierende, offene Ablehnung des Kaisers oder gar für ein Bestreiten seiner Legitimität. Im Gegenteil: Mit Anthemius war zum ersten Mal seit 455 ein Herrscher auf den weströmischen Thron gelangt, an dessen Erhebung die Entscheidungsträger in Ost und West gleichermaßen mitgewirkt hatten. Seine staatsrechtliche Legitimität war unanfechtbar und wurde in den folgenden Jahren selbst von den Gegnern des Kaisers zu keiner Zeit angezweifelt oder auch nur thematisiert. Zwar riefen die Fehler des Anthemius eine oppositionelle Haltung von Teilen der Senatsaristokratie und der Kirche hervor. Diese Ablehnung schlug aber nicht in offenen Widerstand um, sondern erschöpfte sich in distanziertem Abwarten. Andere Teile der Aristokratie, etwa die einflußreiche gens Decia, vor allem aber die großen Senatorenfamilien Galliens, kooperierten auch weiterhin mit dem Kaiser. Mit einer Regierungszeit von mehr als fünf Jahren zählte Anthemius letztlich zu den erfolgreichsten weströmischen Herrschern dieser Periode.

Ausschlaggebend für den Sturz des Anthemius waren schließlich dieselben Kräfte, die seit 455 das Schicksal aller weströmischen Kaiser bestimmt hatten: die Armee und das hohe Offizierskorps unter der Führung des Ersten Heermeisters. ${ }^{15}$ Im Laufe des 5. Jahrhunderts hatte das Amt des Ersten Präsentalen Heermeisters (magister utriusque militiae et patricius) im Westreich einen gewaltigen Bedeutungszuwachs erfahren, während das Kaisertum immer mehr an Gestaltungsspielraum verlor. Vor allem nach 455, in einer Phase

14 Möglicherweise ist die Personalpolitik des Anthemius als Versuch einer Bündelung von Kräften zu verstehen: Durch Sidonius Apollinaris konnte die gallische Aristokratie für den Kaiser gewonnen werden, durch Marcellinus das seit 454 vom Westreich abgespaltene Dalmatien. Die praktizierte, religiöse Toleranz sollte wohl Altgläubige sowie die verschiedenen christlichen Konfessionen ansprechen. In Italien und der Stadt Rom - und damit im Zentrum der kaiserlichen Macht - dominierten jedoch andere Kräfte das politische Geschehen. Der „katholische“ Klerus und große Teile der senatorischen Aristokratie vor Ort erblickten in diesen Versuchen nur einen Affront. Die Fraktionalisierung der weströmischen Eliten, die Anthemius offenbar überwinden wollte, war bereits viel zu weit fortgeschritten.

15 Henning, Krise (wie Anm. 3), 245-260. 
schwacher, rasch wechselnder Kaiser stellte der Heermeister in wachsendem Maße jene Konstante dar, auf die sich das Beziehungsgeflecht der gesellschaftlichen Eliten ausrichtete. Seit es den Heermeistern gelungen war, die Loyalität der Truppen auf die eigene Person statt auf diejenige des Kaisers zu konzentrieren, war ein Regieren gegen sie nicht länger möglich. Mit der Ermordung Valentinians III. scheiterte schließlich der letzte Versuch des Kaisertums, sich aus dieser Umklammerung zu befreien.

Von 456 bis zu seinem Tode im Jahre 472 bekleidete der aus westgotischsuebischem Adel stammende Ricimer das Amt des Ersten Heermeisters. In diesen sechzehn Jahren stürzte bzw. erhob er insgesamt fünf weströmische Kaiser. Ricimer, nicht die Kaiser, war damit der zentrale Machtfaktor des Westreiches. Er residierte in Mailand, im Hauptquartier der italischen Feldarmee, und betrieb von hier aus eine Politik, an deren erster Stelle nicht das Wohl des Imperium, sondern die Bewahrung der eigenen Position stand. Bis zur Erhebung des Anthemius waren alle Versuche der verschiedenen Kaiser, sich Ricimer gegenüber durchzusetzen, gescheitert.

Diese Umstände waren in Konstantinopel, wo zeitweise eine ähnliche Entwicklung gedroht hatte, wohl bekannt. Der Ostkaiser Leo I. bereitete die Entsendung seines Protegés Anthemius deshalb durch intensive Verhandlungen mit Ricimer vor, in deren Mittelpunkt die Sicherung der Machtstellung des Heermeisters sowie - als herausragendes Zugeständnis - seine Anbindung an das neue Herrscherhaus durch eine Ehe mit der Anthemius-Tochter Alypia standen. Ricimer gab sich damit anfänglich zufrieden und unterstützte die Thronerhebung des Anthemius.

Schon bald jedoch verschlechterte sich das Verhältnis beider zueinander. Anthemius war in Begleitung einer großen oströmischen Armee nach Italien gekommen, die Nordafrika von den Vandalen zurückgewinnen sollte. Die Unterstützung durch diese Truppen verlieh ihm aber zugleich eine militärische Machtstellung, gegen die sich Ricimers Möglichkeiten eher bescheiden ausnahmen. Parallel dazu beförderte der neue Kaiser etliche Parteigänger in hohe militärische Positionen und erweckte so den Eindruck, Konkurrenz zur bisher einzigartigen Stellung des Ersten Heermeisters schaffen zu wollen. Ricimer, der zum ersten Mal befürchten mußte, ins machtpolitische Abseits zu geraten, reagierte entsprechend negativ auf diese Entwicklung. Erst das Scheitern des Vandalenfeldzuges im Sommer 468 und der anschließende Abzug der oströmischen Truppen aus Italien eröffnete ihm jedoch die Möglichkeit, aktiv gegen Anthemius vorzugehen. Er ließ nun den Zweiten Präsentalen Heermeister, den vom Kaiser ernannten Dalmater Marcellinus, ermorden und durch einen Offizier seines Vertrauens ersetzen. Im Jahre 470 folgte der offene Bruch mit Anthemius. Ricimer zog sich nach Mailand zurück und 
traf Vorbereitungen für ein militärisches Vorgehen gegen den Kaiser. Nach anfänglichen Verhandlungsversuchen, in deren Rahmen auch die bei Ennodius geschilderte Mission des Bischofs Epiphanius von Pavia zu verstehen ist, eskalierte die Situation im Herbst 471 schließlich zum offenen Bürgerkrieg. Ricimer marschierte auf Rom, schloß Anthemius dort ein und erhob mit Anicius Olybrius einen Gegenkaiser seiner Wahl. Nach neunmonatiger Belagerung fiel die Hauptstadt im Juli 472 schließlich in Ricimers Hand. Anthemius fand dabei den Tod.

Halten wir fest: Als primäre Ursache für das Scheitern des Anthemius ist seine Konfrontation mit dem mächtigen Ersten Heermeister des Westreiches anzusehen. In dieser Hinsicht ist kein Unterschied zwischen seiner Regierungszeit und derjenigen seiner unmittelbaren Vorgänger bzw. Nachfolger in Italien auszumachen. Die griechische Herkunft des Anthemius besaß keinen erkennbaren Einfluß auf die Ereignisse, seine Unterstützung durch Konstantinopel nur insofern, als sie den Ausbruch der Konfrontation deutlich verzögerte.

\section{Zusammenfassung}

Aus den Schriftquellen läßt sich für eine gezielte Verunglimpfung des Anthemius aufgrund seiner griechischen Herkunft als Nicht-Römer nur ein einziges zeitgenössisches Beispiel, nämlich der bei Sidonius Apollinaris überlieferte Fall des Arvandus, finden. Dieses Beispiel aber ist nicht verallgemeinerungsfähig. Ihm gegenüber stehen zahlreiche Belege für die Ablehnung einer solchen Sichtweise durch die senatorische Aristokratie. Die Schilderung des Ennodius dagegen dürfte zwar im Großen und Ganzen auf verläßlichen Informationen beruhen, ist jedoch in ihren Details - und vor allem in der Gegenüberstellung von graecus und catbolicus et Romanus, unter dem Eindruck aktuellen Zeitg eschehens, ex eventu, geformt.

Aus dem historischen Kontext der Jahre um 467 geht hervor, daß Leo und Anthemius selbst die östliche Herkunft des Thronanwärters als Problem bei der Durchsetzung seiner Herrschaft im Westen begriffen. Entsprechende Ressentiments könnten also bei Teilen der westlichen Eliten durchaus vorhanden gewesen sein. Obwohl Anthemius diese Ressentiments durch ungeschickte Maßnahmen sogar noch schürte, scheinen sie auf sein weiteres Schicksal aber keinen erkennbaren Einfluß gewonnen zu haben. Die eingangs formulierte, zugespitzte Frage, ob die bloße griechische Herkunft des Anthemius für seinen Sturz verantwortlich war, kann deshalb klar verneint werden. 
Im Gegenteil: Man kann sogar postulieren, daß es gerade diese Herkunft war, die Anthemius eine vergleichsweise lange Regierungszeit von über fünf Jahren ermöglichte. Die uneingeschränkte Unterstützung des Kaisers durch seinen oströmischen Kollegen nämlich versah ihn mit einer herausragenden Legitimität und anfänglich auch mit umfangreichen Machtmitteln, die in dieser Form kein anderer Westkaiser der Periode aufzuweisen hatte. Möglicherweise war es eben diese unanfechtbare Legitimität des Anthemius, die seine Gegner dazu zwang, andere Angriffspunkte gegen den Kaiser zu suchen, und letztlich seine griechische Herkunft ins Felde zu führen. Entscheidend für seinen Sturz wurde dieser propagandistische Versuch jedoch nicht.

Die tiefe Kluft zwischen den gesellschaftlichen Eliten des alten und des neuen Rom, die John O'Flynn bereits im 5. Jahrhundert für gegeben hält, entstand in einem langsamen, fast schleichenden Prozeß, der die gesamte Spätantike und das Frühe Mittelalter einschloß. Sie wurde anfänglich kaum wahrgenommen, später noch lange Zeit hindurch von einem immer wieder aufscheinenden Gefühl der Zusammengehörigkeit überbrückt, das tatsächliche Entfremdung als bloße Meinungsverschiedenheit interpretierte. In der Agonie des Weströmischen Reiches nach 455 war dieser Proze $\beta$ bereits angelaufen, jedoch weder zu einem Abschluß gelangt noch in seiner zukünftigen Tragweite für die Zeitgenossen überhaupt erkennbar. Kennzeichnend für diese Periode war vielmehr eine Regionalisierung der Oberschicht, in deren Folge beispielsweise der stadtrömischen Aristokratie ihre gallischen Standesgenossen fremder erschienen als die oströmischen. $\mathrm{Da} \beta$ der Westen eines Tages diese gerade erst beginnende, innere Fragmentierung wieder überwinden, mehr oder weniger einheitlich dem Osten gegenübertreten und ihm gar das Römertum absprechen würde, war zur Zeit des Procopius Anthemius gänzlich unvorstellbar. Die Kontinuitätslinie, die O'Flynn von dieser Zeit bis zum „Zwei-Kaiser-Problem“ des Mittelalters zieht, ähnelte - wenn überhaupt - weit mehr einer Zick-Zack-Linie als einer Geraden. 